\title{
COMPRENSIÓN DE LA ENFERMERÍA DESDE LA PERSPECTIVA HISTÓRICA DE FLORENCIA NIGHTINGALE
}

\section{UNDERSTANDING THE NURSING HISTORICAL PERSPECTIVE OF FLORENCE NIGHTINGALE}

\author{
Elizabeth Rocío NúÑEz Carrasco*
}

\begin{abstract}
RESUMEN
El libro Notas sobre enfermería, Qué es y Qué no es, escrito por Florencia Nightingale, cumplió 150 años desde su primera edición, y es sin duda el libro en el cual se dibujaron los primeros lineamientos de la disciplina de enfermería. Este artículo tiene como propósito interpretar desde la experiencia de ser enfermera y desde definiciones contemporáneas de la disciplina, cómo la enfermería ha articulado su desarrollo teórico, y de valorar el impacto de la enfermería en aquella persona llamada a cuidar. Desde las citas del libro fue posible interpretar el avance sistemático de la disciplina de enfermería desde su inicios hasta nuestros días. Se constata cómo el cuidado humano es capaz de movilizar a aquella persona que cuida, inclusive transformándola.
\end{abstract}

Palabras clave: Enfermería, historia, disciplina.

\begin{abstract}
The book Notes on Nursing, What it is and what it is not written by Florence Nightingale, celebrated 150 years since its first edition, and is undoubtedly the book, which drew the first outlines of the discipline of nursing. This paper aims to interpret from the experience of being a nurse and from contemporary definitions of the discipline, how nurses have based their theoretical development, and to assess the impact of nursing on that person called to care. Based on the quotes from the book it was possible to interpret the systematic progress of the discipline of nursing from its inception until today. It is stated how human care is capable of mobilizing the caregiver, transforming it.
\end{abstract}

Key words: Nursing, history, discipline.

Fecha recepción: 23/11/2009 Fecha aceptación: 20/01/2011

\section{INTRODUCCIÓN}

Nacen estas primeras palabras desde la grata necesidad de comprender la enfermería a través de un personaje histórico, como es Florencia Nightingale. Efectivamente, la comprensión de la enfermería a partir de sus ideas que han dado base a los conceptos teóricos de enfermería -llámese disciplina, cien- cia y arte- permite reconocer y reinterpretar nuestros orígenes, observando y reflexionando sobre lo ya construido para iniciar los procesos de transformación.

En la búsqueda de conocer cabalmente estas definiciones, he iniciado una revisión bibliográfica del segundo libro de Florencia Nightingale, de mayor impacto en la enfermería: Notas sobre enfermería, Qué es y Qué no es, el cual fue publicado en diciembre de

\footnotetext{
* Enfermera. Docente Escuela Enfermería Universidad de Santiago de Chile. Estudiante Doctorado en Enfermería. Facultad Enfermería Universidad Andrés Bello. Santiago, Chile, Email: elizabeth.nunez@usach.cl
} 
1859 en Inglaterra y que "en sólo un mes se habían vendido 15.000 ejemplares" (1). En 1861 se realiza una edición de bajo costo económico para la accesibilidad de la clase trabajadora. Este hecho inició la traducción y publicación en todos los idiomas, siendo el capítulo más importante para sus lectores "El cuidado del niño".

\section{CITANDO A FLORENCIA NIGHTINGALE}

La revisión de citas bibliográficas cubrirá dos ámbitos: el descriptivo y el comprensivo. De esta manera, contextualizaremos las citas con los procesos vividos por esta mujer aristócrata del siglo XIX, quien decidió ser enfermera a pesar de la fuerte oposición de su familia. Atrayente es la palabra que describe el título de su libro, Notas sobre enfermería, Qué es y Qué no es; rápidamente el lector comprenderá que el contenido de este libro es acerca de la enfermería. En apariencia una conclusión obvia, pero es importante recordar que este título nace el año 1859, tiempo en donde los contenidos que versan sobre la enfermería sólo estuvieran en el lenguaje oral. Es posible conjeturar que Florencia instala este título como un modo de hacer visible la enfermería.

El título Notas sobre enfermería, Qué es y Qué no es, muestra que la expresión Qué es interroga filosóficamente a la cosa, a la materia, en este caso a este saber que se describe. En otras palabras, la enfermería cobra existencia como concepto teórico, permitiendo, de este modo, la posibilidad de desarrollarse y de construirse. Al definir Qué no es, reconoce sus límites, los cuales son inconfundibles debido a que especifican su característica distintiva de otras esencias o saberes. Este título recoge una intencionalidad, esclarecer que la enfermería existe, por lo que es posible describirla y diferenciarla, es así que la publicación de este texto es una prueba de la convicción de Nightingale por dar a conocer esta verdad. En el prefacio, primera edición 1859, Florencia señala:

"De ninguna manera estas notas tienen la intención de ser una regla de pensamiento por la cual las enfermeras puedan enseñarse a sí mismas a cuidar, ni mucho menos un manual para enseñar a las enfermeras el arte de cuidar. Están dirigidas sencillamente a dar algunas ideas a mujeres que tienen [, $\left.{ }^{*}\right]$ a su cargo personal[,] la salud de otros" (1).

Es posible apreciar el llamado a las enfermeras; afirma, ellas poseen un saber propio, especifica su intencionalidad: no desea ser una profesora o guía, porque las enfermeras ya conocen estas notas y las saben. Afirma que el conocimiento de la enfermería y el saber aplicarlo en manos de las enfermeras se convierte en arte. Sin embargo, Florencia entrega su texto que discurre sobre enfermería a las mujeres, no a las enfermeras. Cabe cuestionar ¿por qué a las mujeres? Más adelante comenta:

\begin{abstract}
“SSaben que en Londres, dos de cada cinco mueren antes de los cinco años? ¿Y que, en las otras grandes ciudades de Inglaterra, mueren casi uno de cada dos? 'La duración de la vida de tiernos niños' (como dice un tal Saturno, que se hizo químico analista) 'es la prueba más sutil' de las condiciones sanitarias. ¿Es necesario que haya tanto sufrimiento prematuro y tanta muerte? ¿O es que la Naturaleza ha destinado a las madres a aprender a tocar el piano [,] que aprender las leyes que ayudan a proteger la descendencia" (1).
\end{abstract}

Luego de esta afirmación, es relevante mostrar el contexto de este libro, el cual fue escrito luego de su experiencia como enfermera de la Guerra de Crimea, donde ella logró, a pesar de la fuerte resistencia de mi-

\footnotetext{
* Correción gramatical a la traducción al español del libro Notes on Nursing: What it is, and what it is not.
} 
litares y médicos, organizar, sistematizar, registrar y evaluar estadísticamente el impacto del cuidado en los soldados enfermos durante la guerra. En 1855 el porcentaje de mortalidad de los soldados es de $42,7 \%$ (2), causado principalmente por enfermedades infecciosas. Tras un año de trabajo de Nightingale "el porcentaje inicial de mortalidad descendió a un 2,2\%, debido al establecimiento de cuidados" (3) tales como: limpieza con agua potable, establecimiento de ropa de cama y lavandería, reorganización de los hábitos alimenticios, mejoramiento del equipamiento hospitalario y habilitación de lugares de recreación de los soldados.

Después de estas vivencias, Florencia vuelve a Inglaterra, dando cuenta de la importancia en la prestación de cuidados (4). Aquello que ella denomina como "el arte de la enfermería" impacta en la salud de las personas, prueba de ello es que observó que las muertes por enfermedades contagiosas imagen frecuente dentro de los hospitales y hogares ingleses de la época- disminuyeron notablemente con los cuidados entregados en Crimea, por lo que se dedujo la necesidad de repetir esta operación en los hospitales y hogares de los ingleses.

Escribe y publica su libro, cuya acción refleja todo el compromiso que asume con la vida de las personas, colocando todos los conocimientos al servicio de las mujeres, ya que son ellas las que cuidan a la descendencia $\mathrm{y}$ a sus familiares enfermos. Es fundamental hacer conciencia de lo complejo que en la época era para las mujeres acceder a conocimientos de cualquier tipo, en especial científicos. Con esta publicación Nightingale, ante los ojos de la modernidad, se convierte en una estratega en salud, ya que observó y dirigió su libro a quienes podían cambiar los procesos de salud-enfermedad de la familia. Las mujeres son su foco de estrategia, porque comprende el rol fundamental de la mujer en los cuidados, siendo necesario educarlas en los conocimientos de la enfermería. ¿Y qué es la enfermería para Florencia Nightingale?:
"Cada día tiene mayor importancia el conocimiento de la higiene, el conocimiento de la enfermería, en otras palabras, el arte de mantenerse en estado de salud, previniendo la enfermedad, o recuperándose de ella. Se le reconoce como el conocimiento que todo el mundo debe tener, distinto del conocimiento médico, propio solamente de una profesión" (1).

Desde estas afirmaciones se desprende que Florencia incorpora el conocimiento de la higiene como parte de la enfermería, dado que el ambiente que observa se encuentra marcado por la suciedad, peste y muerte. Estos hechos propiciaron su énfasis en el ambiente físico más que en el psicológico y social. La enfermería no es sólo un cúmulo de conocimientos, sino es un arte, cuyo foco está centrado en la constante búsqueda de la salud, previniendo la aparición de enfermedades o en la recuperación de ella. Además, la enfermería es un saber que debe ser parte del colectivo para mejorar la calidad de vida de las personas.

Florencia, de una manera clara y precisa señala: este saber es distinto a la medicina. Mientras la medicina diagnostica, la enfermería se dedica a que los cuidados complementen el tratamiento médico, posibilitando una mejor recuperación, e instalando que la enfermería deje de ser un oficio de mujeres, convirtiéndose en una profesión. Volviendo al prólogo del libro:

\footnotetext{
"Si cada mujer, en algún momento de su vida, tiene que ser una enfermera, es decir, tener a su cargo la salud de alguien, qué inmenso y qué valioso sería el fruto de sus experiencias unidas, si cada mujer pensara bien cómo cuidar a otros" (1).
}

Una mujer no es todo el tiempo enfermera, se convierte en enfermera cuando utiliza los conocimientos de la enfermería para la realización de cuidados. En esta acción de cuidar las mujeres se enfrentan a experien- 
cias valiosas que al ser compartidas permiten que los cuidados mejoren, beneficiando a los pacientes. En la conclusión del libro, Florencia señala:

"Pero la enfermería como arte no ha sido tratada aquí por tres razones: 1) que estas notas no pretenden ser un manual de enfermería, como tampoco de cocina de enfermos; 2) que la autora, que ha visto más de lo que puede llamarse enfermería quirúrgica, es decir enfermería manual práctica, tal vez como nadie en Europa, honestamente cree que es imposible aprenderlo en ningún libro y que sólo puede aprenderse bien en las salas de hospital; también ella cree honestamente que la perfección de la enfermería quirúrgica puede verse practicada por una sister chapada a la antigua en un hospital de Londres, como en ninguna otra parte de Europa, y 3 ) mientras que miles mueren a causa del aire contaminado, etc., contando con esta enfermería quirúrgica perfecta, lo contrario es comparativamente raro"(1).

Para Florencia el arte de la enfermería se encuentra en la práctica, en el contacto con quien se cuida. En su relato se constata el ímpetu, el cuidado debía ser un acto reflexivo, porque a pesar de realizar cuidados en las salas de hospital de manera perfecta a la luz de la técnica, las personas estaban muriendo porque las condiciones ambientales no eran aptas para la recuperación de su salud. Es curioso evidenciar que el único lugar donde Florencia pudo enseñar el arte de cuidar, así como lo entendía ella, fue en la Guerra de Crimea. Incluso siendo la creadora de la Escuela de Enfermería, ella no realizaba docencia ya que se presentaba físicamente imposibilitada, además sentía que su trabajo no había tenido un impacto en las enfermeras, describiéndolo así:

"Mis teorías no han suscitado interés entre las mujeres. Las que fueron conmigo a Crimea no aprendieron nada de mí, y ninguna [...] ha sacado las lecciones de la guerra" (5).

\section{ENFERMERÍA CONTEMPORÁNEA}

Luego de la revisión del libro de Florencia Nightingale, es posible describir los conceptos de disciplina, ciencia y arte de la enfermería desde algunos referentes contemporáneos, para visualizar cómo estos conceptos han evolucionado desde los inicios de la enfermería moderna.

Los requisitos que constituyen una disciplina son: "especificar su lugar apropiado en la sociedad y definir los parámetros de conocimientos necesarios para su práctica" (6). La disciplina para dar respuesta a esta afirmación debe "explicar sus tradiciones históricas, sociales, y filosóficas para entender su conexión a otros en el campo de la epistemología, y proporcionar un contexto significativo para sus métodos de investigación y áreas adecuadas para la práctica" (6).

Validado este concepto, es posible establecer que el realizar un recorrido histórico es estar dentro de la disciplina comprendiendo y conectando el aporte de la tradición cultural de la enfermería a nuestra contemporaneidad. Florencia, al publicar su libro, deja clara constancia que la enfermería existe porque posee conocimientos propios, otorgándole un dominio en el campo del saber, y que la razón de su existencia es la búsqueda de la salud de las personas en un proceso continuo de prevención, mantención y recuperación de la salud. Jean Watson comprende que la disciplina:

\footnotetext{
"Es el cuerpo de conocimientos, los valores, lo ético, las teorías, y los límites de la estructura del conocimiento, los fundamentos meta-narrativos de la profesión, los cuales informan y guían la práctica actual de esas teorías, la ética, los valores, y el conocimiento... el conocimiento disciplinario es el conocimiento que viene de las raíces de la historia de enfermería, de las tradiciones, de la herencia a través del tiempo, así como de las teorías y del conocimiento existente"(7).
} 
La disciplina de la enfermería, que está constituida por los conocimientos de la enfermería, por su valores, reflexión ética, por sus fundamentos profesionales, se retroalimenta cuando la enfermera realiza la acción de cuidar, y es esta experiencia la que -a lo largo de los años- se ha plasmado en libros, revistas y tradición oral. Luego es compartida por el colectivo de enfermeras, quienes son las que validan esta herencia. De este modo, la "disciplina de enfermería" valida a la "enfermera(o)" en una continua retroalimentación entre la profesión y la disciplina. Son las enfermeras y enfermeros quienes al realizar cuidados definen cómo se organizarán estos conocimientos.

Jean Watson agrega: "La disciplina de enfermería también convoca un sentido de disciplina personal, que es el desarrollo ontológico y espiritual de las enfermeras mismas, el cultivo de los niveles más profundos de nuestra propia humanidad, del sufrimiento humano, y del proceso de sanación"(7). La incorporación del concepto de "disciplina personal" acuñado por Watson muestra que es necesario que la enfermera, para lograr un mejor desempeño en el cuidado humano, desarrolle la empatía para comprender a las personas en su proceso de sufrimiento, fortaleciendo su crecimiento espiritual.

Asistir a una guerra -como lo hizo Florencia Nightingale-, en donde vivió el abandono, el dolor, el sufrimiento y la muerte humana en forma directa (8), la convirtió en una mujer que a pesar de su condición femenina-sabemos que en su época fue un obstáculo- lideró las reformas sanitarias dentro de los hospitales que actualmente conocemos (9). El recuerdo y las vivencias recogidas en la guerra la hicieron ser persistente en la tarea de cuidar a otros, marcándola en su historia de vida, así lo demuestran sus palabras grabadas en voz por Edison (10) en 1890, en donde ruega a Dios por sus camaradas de Balaclava.

El nacimiento de la palabra ciencia es un lenguaje relativamente reciente en la enfer- mería, dado que en 1950 se publicó la primera teoría de enfermería: "La teoría Interpersonal de la Enfermería de Hildegaard Pepplau, primera enfermera que escribe y publica una visión de enfermería después de casi un siglo de silencio, desde Florencia Nightingale" (11). Luego de este impulso, la creación de teorías de enfermería, desarrolladas fundamentalmente en el mundo anglosajón, se multiplica de un modo extraordinario. En menos de 60 años existen innumerables textos escritos, marcos conceptuales, modelos y teorías donde las enfermeras han podido plasmar sus visiones sobre la enfermería.

La "ciencia de la enfermería" durante largo tiempo estuvo marcada por la búsqueda de "conocimiento empírico, factual, descriptivo y finalmente dirigido a desarrollar explicaciones abstractas y teóricas" (11). Este marcado paradigma no fue capaz de dar respuesta al complejo escenario que es cuidar a un ser humano. Es por esta razón que la enfermería ha transitado en búsqueda de un paradigma en donde "se formulen las preguntas de investigación desde los patrones más pertinentes a la naturaleza del fenómeno en estudio y aplicar los métodos que permitan construir conocimiento" (11).

Elizabeth Marhart define la "ciencia de la enfermería" como:

\footnotetext{
"Un conocimiento abstracto y sustantivo que describe el fenómeno único de Enfermería que le concierne, la naturaleza integral de los seres humanos unitarios y sus ambientes. La creación de este conocimiento ocurre a través de la síntesis así como de los métodos cualitativos y cuantitativo de indagación (...) la ciencia de Enfermería basada en la práctica es la que usa conocimiento creativo e imaginativo que promociona la salud y el bienestar de toda la gente" (12).
}

Esta definición muestra dos miradas de la ciencia en enfermería, por una parte señala que la ciencia de enfermería es la generación de conocimiento abstracto, que visualiza a la 
persona como un ser único relacionado con su ambiente, y la segunda, aquella en donde el conocimiento creativo e imaginativo está utilizado en la práctica que posee como finalidad promocionar salud y bienestar a las personas, siendo los métodos cualitativos y cuantitativos los que permitan indagar en este conocimiento. Al parecer, Florencia Nightingale se refiere a ese conocimiento que se genera cuando se está sintiendo al otro en su proceso único de salud, es el conocimiento basado en la práctica.

En varias oportunidades del relato Florencia enfatiza, la enfermería es un arte, describiéndolo como aquello que se genera con el otro, cuando se vivencia el proceso de cuidar, cuando se crea la mejor condición de cuidado para ese ser que es único, es allí donde muchas veces su relato emerge con mayor intensidad y convicción, convirtiéndose en llamado potente para quienes han elegido la inquietud por cuidar.

Reed, Sheares, Nicoll señalan que "el arte de la enfermería es inseparable de la ciencia de la enfermería" (13), porque el arte y la ciencia se generan cuando se está cuidando al otro. Chinn \& Watson expresan "en la práctica de Enfermería, el saber estético es expresado a través de aquellos actos que sirven para transformar una experiencia desde lo que es en el presente para lo que es posible que sea en un futuro. La enfermera moviliza, a través de la experiencia artística, a la persona desde los límites de la enfermedad y desafíos para la salud hacia experiencias de curación/sanación y nuevas posibilidades" (11).

\section{CONSIDERACIONES FINALES}

En conclusión, es posible visualizar cómo el hilo conductor de la historia de la enfermería a través de Florencia Nightingale otorga comprensión al desarrollo teórico de la enfermería. Esta forma de comprensión permite inferir que la disciplina de enfermería son conocimientos que han sido liderados por mujeres a través del lenguaje oral, y que fue develado por Florencia Nightingale a través de su segundo libro. Significa entonces, que es ella quien escribe y describe el propósito de este cúmulo de conocimientos, añadiendo además que el foco de la enfermería es la salud de las personas, y que es el cuidado del ambiente (14) la forma para la prevención o recuperación de la salud.

Ciencia, palabra que otorga un posicionamiento dentro del concierto del conocimiento, es posible comprenderla desde los escritos de Florencia, como un ordenamiento teórico de la realidad del cuidado de las personas, el cual debía ser riguroso. Importante es recalcar que la realidad observada por Florencia fue de personas hacinadas en sus hogares con niños que morían por enfermedades infecciosas, al igual que las personas que acudían a los hospitales. En este escenario despliega una construcción de cómo la Enfermería, a través del cuidado en la ventilación, calor, alimentación, ruido e iluminación mejora la salud de la personas. Ciento cincuenta años después es posible visualizar que esta construcción teórica sigue siendo la base del cuidado de la personas en los espacios hospitalarios.

Arte, palabra muchas veces invocada por Florencia, es posible reflejarla en ese espacio de tiempo como aquello que se encuentra en la "acción de cuidado", en la conversación con la persona, en el tiempo justo para aplicar cierta técnica, en la mirada, en la energía entre la enfermera y la persona cuidada.

Nightingale posee numerosos detractores que señalan su conducta neurótica y compulsiva, además de su cercanía con militares y religiosas (15), sin embargo estos elementos dibujaron su contexto y mundo de vida (16), que indudablemente la marcaron. Asimismo, estos paradigmas, que a juicios de sus detractores son limitantes, no impidieron que esta mujer fuera acallada para escribir, hablar, discutir y debatir sobre cómo la enfermería impacta positivamente en las 
personas. Florencia Nightingale, definitivamente fue una mujer transgresora, que movida por el cuidado hacia las personas se hizo cargo de esta convicción, escribió, publicó y gestionó en las más altas esferas políticas formas para asegurar el cuidado de las personas.

Sin lugar a dudas, es la madre de la enfermería, en lo personal he querido transmitir que el cuidado humano moviliza, generando una energía de la cual no se puede huir, incluso transformando al que otorga cuidado, así lo evidencian las historias de vida de esta mujer y de miles de mujeres que han seguido el camino del cuidado. Un ejemplo, Helen Howitt, enfermera canadiense, que deja su país para liderar los procesos de profesionalización de enfermeras en Latinoamérica de manera incansable, caracterizada por su estilo rígido pero muy humano (17). En Chile Gladys Peake, quien luego de estudiar un magíster en Educación para Enfermería en Canadá lidera como primera directora la Escuela de Enfermería de la Universidad de Concepción (18). Posteriormente, en la década de los años cuarenta y cincuenta organiza todos los servicios de enfermería del país, convirtiéndose en la enfermera jefe del Departamento de Enfermería de Chile, simultáneamente en conjunto con otras enfermeras organiza el Colegio de Enfermeras de Chile (19). Su estilo, una mujer en una búsqueda permanente de mejoras a través del conocimiento, altamente rigurosa con sus pares, pero con una fuerte convicción de entrega frente a las personas que requerían de cuidado.

Este paradigma de mujer-enfermera ha marcado numerosas generaciones, indudablemente ahora asistimos a una transición paradigmática del ser enfermera-mujer, cuyo proceso está dado por los cambios sociales, económicos y culturales de la mujer. Mas existe una esencia, que es mantenida a través de la historia de la enfermería, cada gesto en el otro, cada llanto, cada alegría, cada angustia de esa persona que requiere cuidado moviliza a las enfermeras y enfermeros para la acción, y este recorrido permite comprender el valor de la obra de Florencia Nightingale, en cuyo inicio identificó los primeros fundamentos propios y singulares de la disciplina (20) y profesión, y que aún permanecen vigentes.

\section{REFERENCIAS}

1. Nightingale F. Notes on Nursing: What it is and what is not. En J. Castro. $2^{\mathrm{a}} \mathrm{ed}$. Notas sobre enfermería Qué es y que no es. México: Salvat Editores (Original publicado en 1946). 1991.

2. Hanzeliková A, Carabaño M, Torresano B, García M. Florencia Nightingale y el Contexto Histórico Sociocultural de su Epoca. Cultura de los Cuidados. 2005; 9(18): 24-33.

3. Donahue P. Historia de la Enfermería. 2a ed. España: Grafos, S. A. Arte sobre papel; 1985.

4. Woodham-Smith, C. Florencia Nightingale. $2^{\text {a }}$ ed. Buenos Aires: Losada Editorial; 1957.

5. Attewell A. Florencia Nightingale. Perspectivas. 1998; 28(1): 173-189.

6. Grossman M, Hooton M. The significance of the relationship between a discipline and its practice. Journal of Avanced Nursing. 1993; 18(6): 866-872.

7. Fawcett, J. The Nurse Theorists: 21stCentury Updates-Jean Watson. Nurs Sci Q. 2002; 15(3): 214-219.

8. Florence Nightingale Museum. Crimea War. Hallado en: http://www.florencenightingale.co.uk/cms/. Acceso el 7 julio de 2009.

9. Internacional Council of Nurses. Notes on Nursing A guide for Today's Caregivers. $1^{\text {a }}$ ed. España: Baillière Tindall Elsevier; 2009.

10. Florence Nightingale. The Cylinder Recording. Hallado en: http://www. countryjoe.com/nightingale/cylinder. 
htm. Acceso el 7 julio de 2009.

11. Rivera MS. Formas de conocer en Enfermería: el sustento teórico de la práctica profesional. Horizonte de Enfermería. 2003; 14(1): 21-32.

12. Barret A, Daly J, Mitchell G, Toikkanen $\mathrm{T}$, Miller B, Zanotti R, Takahasi $\mathrm{T}$ et al. What is nursing science? An international Dialogue. Nurs Sci Q. 1997; 10(1): 10-13.

13. Royse M, Newton S. Nursing Education Perspectives. 2007; 28(5): 263-267.

14. Marriner-Tomey A, Alligood MR. Modelos y teorías en enfermería. $3^{a}$ ed. España: Elsevier Sciencie; 2003.

15. Webb V. Florence Nightingale: the making of a radical theologian. $1^{\text {a }}$ ed. United States of America: Chalice Press; 2002.

16. González T. Florencia Nightingale Profesionalización de los cuidados desde una perspectiva de la antropología feminista. Cultura de los Cuidados. 2005; 9(17): 3340.

17. Velandia AL. Helen Howitt: una semilla canadiense en la enfermería latinoamericana. Av Enferm. 2009; 27(1): 93-101.

18. Flores R. Historia de la Enfermería en Chile. Sintesis de su evolución educacional. $1^{\text {a }}$ ed. Santiago de Chile: Universidad de Chile; 1965.

19. Colegio de Enfermeras de Chile. Enfermeras destacadas. Hallado en http:// www.colegiodeenfermeras.cl/info. asp?ob=3\&id=30. Acceso el 25 julio de 2010.

20. Johnson B, Webber P. An Introduction to Theory and Reasoning in Nursing. $1^{\mathrm{a}}$ ed. United States of America: Lippincott; 2001. 